\title{
Bacteremia Pattern in Febrile Neutropenia among Adults Cancer Patients Receiving Chemotherapy in an Australia Regional Hospital
}

\author{
Tunde Maiyaki Ibrahim ${ }^{1,2, *}$, Christine Pang ${ }^{1}$ \\ ${ }^{1}$ Department of Medicine, Goulburn Valley Base Hospital, Graham Street Shepparton, Victoria, Australia \\ ${ }^{2}$ Clinical Rural School of Medicine, The University of Melbourne, Graham Street, Victoria, Australia
}

Copyright $\bigcirc 2017$ by authors, all rights reserved. Authors agree that this article remains permanently open access under the terms of the Creative Commons Attribution License 4.0 International License

\begin{abstract}
Background: Febrile neutropenia (FN) remains one of the most concerning complications of cancer chemotherapy, and is a major cause of morbidity and mortality, consuming significant healthcare resource .This audit was carried out to determine the pattern of microbial pathogens responsible for $\mathrm{FN}$ in our institution as this will result in the appropriate choice of empirical antibiotic(s) for treatment of FN in the future. Methods: This is a retrospective audit of adult patients with cancer admitted with FN post chemotherapy in Goulburn valley base hospital, Shepparton, Australia between 2011- 2013.Only patients who met the diagnostic criteria of FN as defined by the Infectious diseases society of America (IDSA) were included in the audit. Results: Twenty six patients presented with 31 episodes of FN between January 2013 and January 2014. 65 blood cultures (BC) were obtained with an average of 2.5 sets of $\mathrm{BC}$ per patients, $10.8 \%$ of these were positive. $66.7 \%$ of the BC yielded Gram Positive Cocci (GPC) $(50 \%$ of which were coagulase positive staphylococci), $33.7 \%$ of the $\mathrm{BC}$ yielded gram negative bacilli (GNB) and 2 yielded multiple organisms. The mean neutrophil count on admission for all the 26 patients was $0.303 \pm 0.25 /$ ul. Those with positive BCs had significantly higher hs-CRP with the mean value of $223.83 \pm 94.27 \mathrm{mg} / \mathrm{l}$, compared to those with negative BCs with $89.37 \pm 79.53$ $\mathrm{mg} / \mathrm{l}$ (t-test $=-3.489, \mathrm{p}=0.002)$. The most common malignancies were hematological and breast cancers with 8 patients each. The presumed focus of infection was mostly in the respiratory tract accounting for $42 \%$ of the cases. $30.8 \%$ of the 26 patients with FN had central venous access device(CVAD) in-situ but all had negative BCs however the odd ratio of developing $\mathrm{FN}$ if CVAD is present is high at 4.3(95\% CI 1.01-18.0). Conclusions: the prevalence rate of bacteremia in post chemotherapy $\mathrm{FN}$ in our center is relatively low and GPC are the most commonly isolated organisms. Our study also support the notion that hs-CRP may be a sensitive biomaker of bacterial infection in cancer patients with post chemotherapy $\mathrm{FN}$ as it is significantly
\end{abstract}

higher in those with positive BC.

Keywords Febrile Neutropenia, Cancer Patients, Blood Cultures, Chemotheraphy

\section{Background}

Febrile neutropenia (FN) remains one of the most concerning complications of cancer chemotherapy, and is a major cause of morbidity and mortality, consuming significant healthcare resource[1]. Most patients with post chemotherapy FN have no infectious cause. Among those with positive blood cultures(BC) microbiological detection rates by standard blood cultures vary depending on whether patients have received prophylactic antibiotic or have a central venous catheter (CVC) [2,3]. The prognosis is worst in $\mathrm{FN}$ patients with proven bacteremia, with mortality rates of around $18 \%$ in Gram-negative (GN) and $5 \%$ in Gram-positive (GP) bacteraemia [3] This audit was carried out to determine the pattern of microbial pathogens responsible for $\mathrm{FN}$ in our institution as this will result in the appropriate choice of empirical antibiotic(s) for treatment of $\mathrm{FN}$ in the future.

\section{Material and Methods}

This is a retrospective audit of adult patients with cancer admitted with febrile neutropenia post chemotherapy in Goulburn valley base hospital, Shepparton, Australia between 2011-2013.Only patients who met the diagnostic criterial of FN as defined by the Infectious Diseases Society of America (IDSA),[4] were included in the audit; Patients would have had 1) Chemotherapy within preceding 14 days, 2) Fever with temperature of $>38.5$ or $>38.0$ lasting for more than $1 \mathrm{hrs}$, and 3) Absolute neutrophil count of $<0.5 /$ ul or 
$<1.0 /$ ul with decline within $48 \mathrm{hrs}$ of admission were included in this audit.

The records of the patients were assessed on chartview computer program, and the leucocyte count, high sensitive C-reactive protein (hs-CRP), and blood culture (BC) results were assessed on the Labtrack computer sites.

\section{Results}

Twenty six patients presented with 31 episodes of FN between January 2013 and January 2014. 65 blood cultures (BC) were obtained with an average of 2.5 sets of BC per patients, $10.8 \%$ of these were positive. $66.7 \%$ of the $\mathrm{BC}$ yielded gram positive cocci (GPC), 50\% of which were coagulase positive staphylococci, the detail is shown in table 1. 50\% of GPC were methicilline resistant, and the only staphylococcus aureus and Group $C$ beta hemolytic streptococcus isolated were also resistant to penicillin. Thirty three point seven percent $(33.7 \%)$ of the BC yielded gram negative bacilli and 2 yielded multiple organisms.
Table 1. Microbial isolates from blood cultures of cancer patients with febrile neutropenia

\begin{tabular}{|c|cc|}
\hline Isolated organisms & \multicolumn{2}{|c|}{ Number of isolate (\%) } \\
\hline Coagulase positive staphylococcus & 3 & $(33.3)$ \\
\hline Staphylococcus aureus & 1 & $(11.1)$ \\
\hline Streptococcus Group C beta hemolytic & 1 & $(11.1)$ \\
\hline Enterococcus Faecum & 1 & $(11.1)$ \\
\hline Escherichia Coli & 1 & $(11.1)$ \\
\hline Pseudomonas Spp & 1 & $(11.1)$ \\
\hline Acromonas Sobria & 1 & $(11.1)$ \\
\hline
\end{tabular}

The mean neutrophil count on admission for all the 26 patients was $0.303 \pm 0.25 / \mathrm{ul}$ and the mean hs-CRP in all patients was $113.39 \pm 99.48 \mathrm{mg} / \mathrm{l}$. Those with positive $\mathrm{BC}$ had significantly higher hs-CRP with the mean value of $223.83 \pm 94.27 \mathrm{mg} / \mathrm{l}$, compared to those with negative $\mathrm{BC}$ with $89.37 \pm 79.53 \mathrm{mg} / \mathrm{l}$ (t-test $=-3.489, \mathrm{p}=0.002$ ). The details of the types of malignancies is shown in figure 1, the most common malignancies for which the patients received chemotherapy were hematological and breast cancers with 8 patients each.

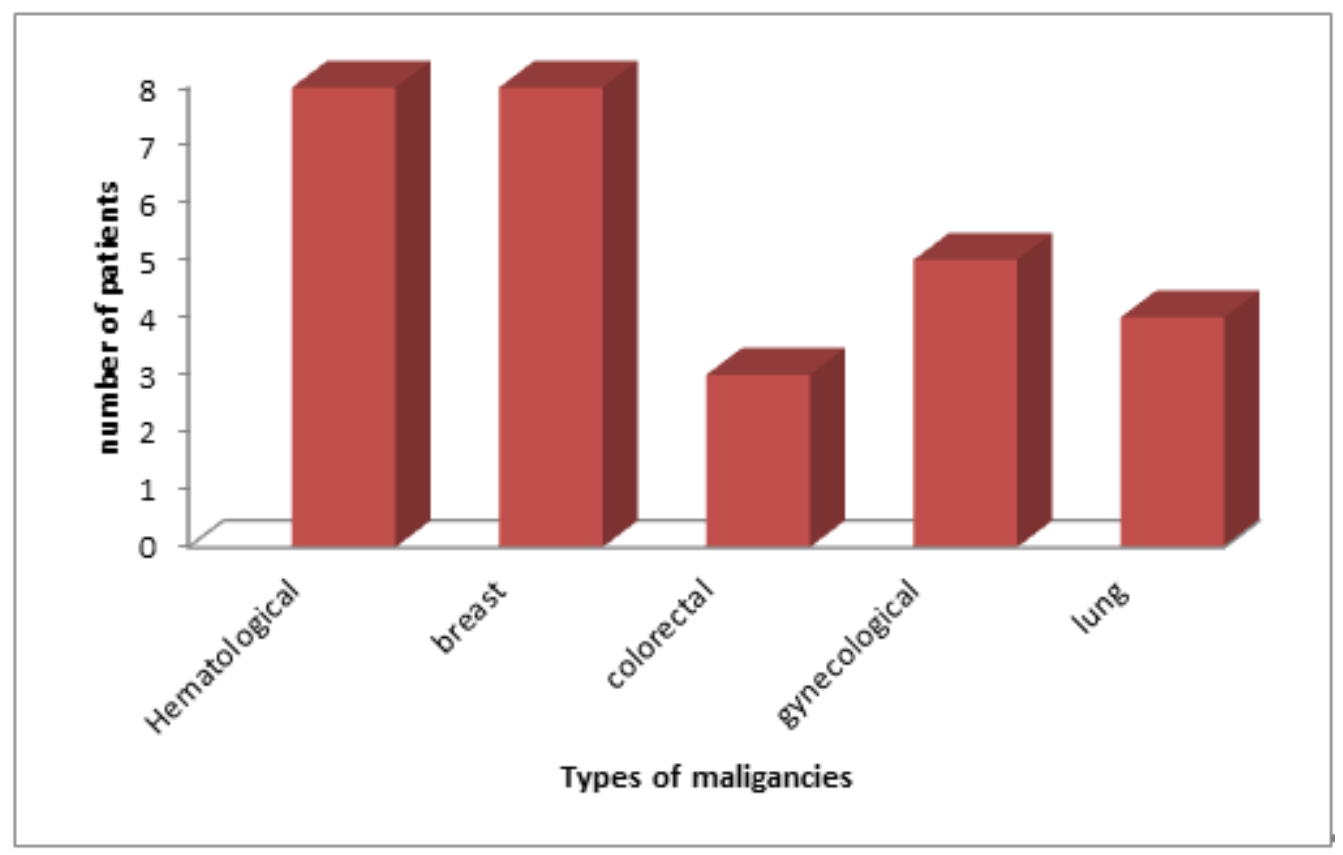

Figure 1. Types of malignancies for which patients were recieving chemotherapy 


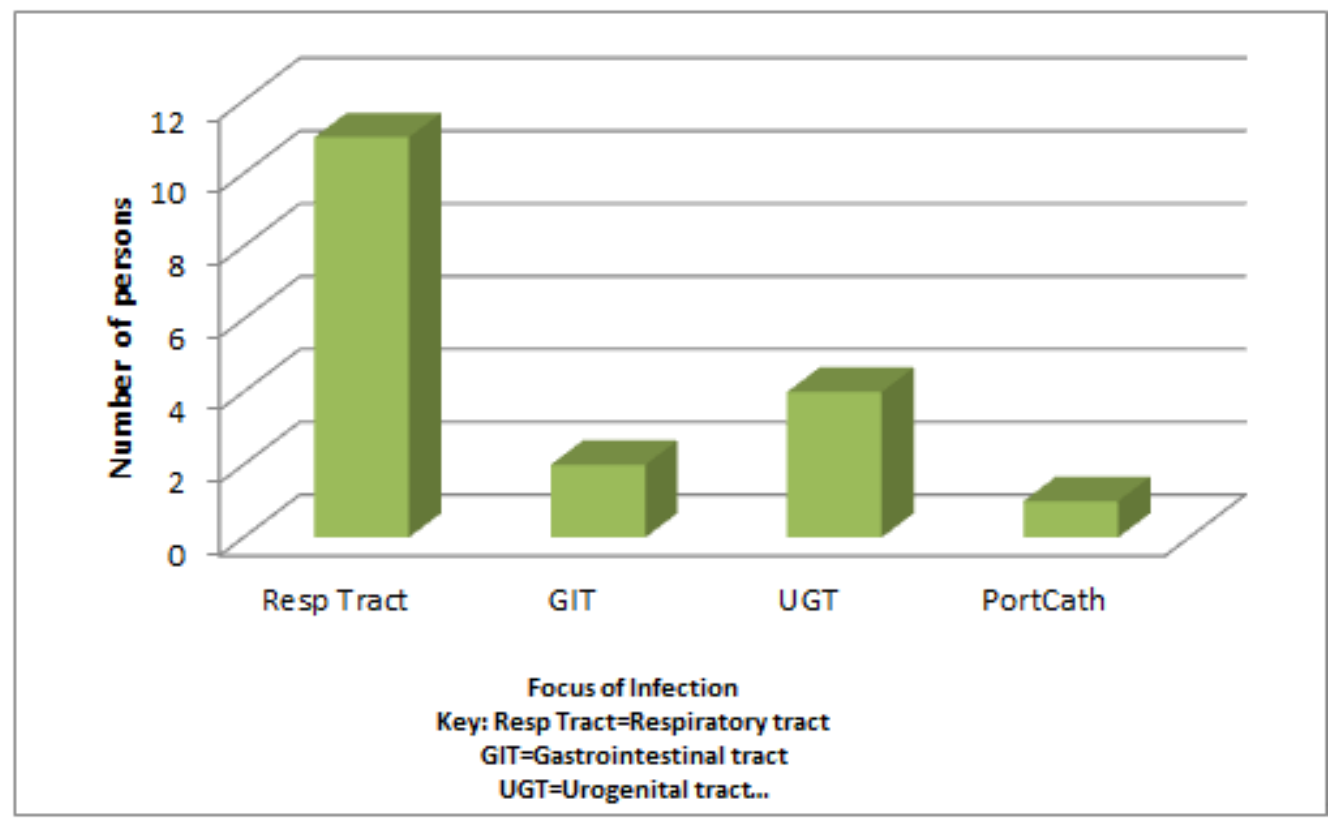

Figure 2. Focus of infection in patients with febrile neutropenia post chemotherapthy

The presumed focus of infection was recorded in 18 patients with 11 patients having the respiratory tract as the most suspected focus the detail is shown in figure 2. 5 (30.8\%) of the 26 patients with FN had central venous access device(CVAD) in-situ from which $\mathrm{BC}$ was obtained and one catheter tip cultured but all returned negative, however the odd ratio of developing FN if CVAD is present is high at $4.3(95 \%$ CI $1.01-18.0)$.

\section{Discussion}

Fever occurs frequently during chemotherapy-induced neutropenia, but majority of post chemotherapy FN are not due to infectious causes. Only $20-40 \%$ of FN episodes in cancer patients receiving chemotherapy are due to bacteremia $[3,5]$, this prevalence is much higher than the $10.8 \%$ observed in this audit. Different centers experience different prevalence rates of FN probably because of the lack of unanimity in the diagnostic criteria, the prevalence of the routine use of prophylactic antibiotics and granulocyte colony stimulating factors, and the use of CVAD.

The trend of the microbial pattern of blood cultures in FN is changing worldwide with Gram positive organisms been the most common in developed countries, while in developing countries GN organisms are still the leading causes of FN. One study from Malaysia reported that $66 \%$ of FN in their cancer patients receiving chemotherapy was due to GN organisms. [1, 4, 5, 6, 7]

The finding from this audit is similar to those in other developed countries including one from Darwin in Australia,[8] where most of the isolated organisms are gram positive cocci. And majority of these are coagulase negative staphylococci. The raising prevalence of GP organisms is said to be due to the increasing use of plastic intra vascular devices, local environmental condition and the wide use of prophylactic antibiotics [9, 10]. Viscoli et al. [9] observe that the presence of CVAD and infection around them are predictors of GP bacteremia in cancer patients with granulocytopenia who had fever. Similarly Seifert et al. [10] reported that $50 \%$ of the 18 cancer patients in their study with CVAD who had post chemotherapy FN had coagulase negative staphylococci bacteremia. The frequencies of GP, GN and polymicrobia organisms found in this audit is like those reported in earlier studies. $[6,11]$ Significant number of the GPC isolated in this audit like those in earlier reports. $[6,7,9,12]$ were coagulase negative staphylococci $(50 \%)$, and about half were MR (50\%). The increase prevalence of MR staphylococci is said to be due to increase use of prophylactic antibiotics especially in non-febrile neutropenic patients. $[1,6,9,12]$ E.coli and pseudomonas spp are the most common GN isolates in our center like in earlier reports. $[1,3,12]$ Factors that might predict GN bacteremia included the presences of shock in the patients and prior use of quinones as prophylaxis in afebrile granulocytopenia.[ 9] With the low positivity rate of $\mathrm{BC}$ in patients with $\mathrm{FN}$ in most centers other biomarkers of bacteremia are now been considered as surrogate for bacteremia in these patients. These include serum highly sensitive $\mathrm{C}$ reactive protein (hs-CRP), procalcitonin (hs-PCT, and interleukin-6 (IL 6) levels. High levels of hs-CRP and hs-PCT levels have been reported to be sensitive in detecting bacterial infection in cancer patients with FN (67\% and 82\% respectively).[12] Von Lilienfeld- Toal et al. [13] also reported that hs-PCT of $>1.8 \mathrm{ng} / 1$ and IL6 $>942 \mathrm{pg} / \mathrm{l}$ are highly suggestive of bacteremia in those cancer patients with post chemotherapy FN. The findings in this audit support these propositions as the CRP was more significantly higher in those with positive BC. The clinical implication of this is that high CRP above certain level is highly suggestive of bacteremia in those with 
FN and can be used as a surrogate marker of bacteremia in patients with culture negative FN. In conclusion, the prevalence rate of bacteremia in post chemotherapy $\mathrm{FN}$ in our center is relatively low with GP organisms that are mostly MR been the most common organism isolated in the BC. Our study also support the notion that hs-CRP may be a sensitive biomaker of bacterial infection in cancer patients with post chemotherapy $\mathrm{FN}$ as it is significantly higher in those with positive $\mathrm{BC}$.

\section{REFERENCES}

[1] de Naurois J, Novitzky-Basso I, Gill M J , Marti Marti F, Cullen M H, Roila F. Management of febrile neutropenia: ESMO clinical practice Guidelines. Ann Oncol. 2010; 21: v252-v256.

[2] Wang X J, Wong M, Hsu L Y, Chan A. Cost associated with febrile neutropenia in solid tumor and lymphoma patients- an observation study in Singapore. BMC Health Serv Res.2014;14(1):434

[3] Feld, R. Bloodstream infections in cancer patients with febrile neutropenia. Int J Antimicro Agents. 2008; 32: S30-S33.

[4] Freifeld AG, Bow EJ, Sepkowitz KA, Boeckh MJ, Ito JI, Mullen CA, Raad II, Rolston KV, Young JH, Wingard JR Clinical Practice Guideline for the Use of Antimicrobial Agents in Neutropenic Patients with Cancer: 2010 Update by the Infectious Diseases Society of America Clinical Infectious Diseases 2011; 52(4):e56-e93

[5] Baskaran ND, Gan GG, Adeeba K, San I C. Bacteremia in patients with febrile neutropenia after chemotherapy at a university medical centre in Malaysia. International journal of infectious diseases. 2007; 11(8):513-517.

[6] Klastersky J, Ameye L, Maertens J, Georgala A, Muanza F, Aoun M, Ferrant A, Rapopport B, Rolston K, Paesmans M. Bacteremia in febrile neutropenia cancer patients. Int $\mathrm{J}$
Antimicrob Agents. 2007; 30:S51-S59.

[7] Ramphal R. Changes in the etiology of bacteremia in febrile neutropenic patients and the susceptibilities of the currently isolated pathogens. Clin Infect Dis 2004; 39(Suppl 1):S2531.

[8] Healey T, Selva-Nayagam S. Retrospective review of febrile neutropenia in the Royal Darwin Hospital, 1994-99.Intern Med J. 2001; 31(7):406-412.

[9] Viscoli C, Bruzzi P, Castagnola E, Boni L, Calandra T, Gaya H, Meunier F, Feld R, Zinner S, Klastersky J. et al Factors associated with bacteraemia in febrile, granulocytopenic cancer patients. The International Antimicrobial Therapy Cooperative Group (IATCG) of the European Organization for Research and Treatment of Cancer (EORTC). Eur J Cancer. 1994; 30A (4):430-437.

[10] Seifert H, Cornely O, Seggewiss K, Decker M, Stefanik D, Wisplinghoff H, Fätkenheuer G. Bloodstream Infection in Neutropenic Cancer Patients Related to Short-Term Nontunnelled Catheters Determined by Quantitative Blood Cultures, Differential Time to Positivity, and Molecular Epidemiological Typing with Pulsed-Field Gel Electrophoresis J. Clin. Microbiol. 2003; 41(1):118-123.

[11] Morris P G, Hassan T, McNamara M, Hassan A, Wiig R, Grogan L, Breathnach O S, Smyth E, Humphreys H. Emergence of MRSA in positive blood cultures from patients with febrile neutropenia--a cause for concern. Support Care Cancer. 2008; 16(9):1085-1088.

[12] Aimoto M, Koh H, Katayama T, Okamura H, Yoshimura T, Koh S, Nanno S, Nishimoto M, Hirose A, Nakamae M, Nakane T, Nakamea H, Kakeya H, Hino M. Diagnostic performance of serum high - sensitivity procalcitonin and serum $\mathrm{C}$-reactive protein test for detecting bacterial infection in febrile neutropenia. Infection.2004;

[13] von Lilienfeld-Toal $M$, Dietrich $M$ P, Glasmacher A, Lehmann L, Breig P, Hahn C Schmidt- Wolf IG, Marklein G, Schroeder S, Stuber F. Markers of bacteremia in febrile neutropenia patients with hematological malignancies: prolcalcitonin and IL-6 are more reliable than C-reactive protein. Eur J Microbiol Infect Dis.2004; 23(7):539-544. 